\title{
EDUCATIONAL EVALUATION AND ANALYSIS OF STATISTICAL TECHNIQUES
}

\author{
Dr. Daruri Venugopal, Professor, OPJS University, Churu, Rajasthan, India, \\ profdarurivg.edu@gmail.com
}

\begin{abstract}
In education evaluation plays an important role in the teaching-learning process. In Evaluation analysis it contributes to formulation of objectives. Structuring of learning experiences and assessment of learner performance. It is very useful to bring improvement in teaching and learning process. It provides accountability to the society, parents, and to the education Systems. Educational and Statistical Evaluation scrutiny provides the accurate results as approved judgments In evaluation process good evaluation always coordinated by Validity, Reliability, practicability, fairness, and Usefulness. In Statistical Analysis process we can identify the Quantitative Data, Continuous and Discrete Data, Qualitative, primary and Secondary Data. In Educational Evaluation Statistical Techniques plays a vital role Standard graphic forms will always provide evaluation confirmation in easy understanding method in the form of
\end{abstract} Graphical representations.

Keywords — assessment of learner, curriculum, Evaluation, learner performance, practicability, Reliability, Scrutiny.

\section{INTRODUCTION}

Evaluation is one of the most important stage in teachinglearning process. It is inevitable in classroom teaching as it is in all fields of activity when judgements need to be made, however simple or complex the consideration involved. The need of evaluation is important factor to the teachinglearning approaches that even hasty consideration seems to indicate the advantages of a systematic use of planned evaluation. Evaluation helps faculty to make better evaluative judgments. Majorly it fulfill class room objectives. Provides the diagnose learning difficulties of students. It determines readiness for new learning experiences, helps to prepare reports of pupils' progress and also to assist students in their problems of adjustment.

The Evaluation process confirm the capacity to work and modifies the learner's behavior. There term evaluation conveys several meaning in education and in psychology. Evaluation is both qualitative and quantitative. In Encyclopedia of Education Research, Stone states, “ Evaluation is a relatively new technical term introduced to designate a more comprehensive concept of measurement than is implied in conventional tests and examination".

\section{BASIC FEATURES OF EVALUATION}

Fairness : Evaluation methods must be fair to all students. This can be possible by accurate transparent of range of expected behaviors as desired by the course objectives. To keep fairness in evaluation, it is also desired that students must known the system of Evaluation in Education Sector. It means that students should be provided information about evaluation such as nature of the factors, parameters on which they are to be examined.

Practicability: Evaluation system should be practical and efficient It may be an ideal procedure of evaluation but may no be put into practice.

Usefulness: By knowing the student strength and weakness, students can think of further improvement, Evaluation should suggest all the needful requirements for their improvement.

Reliability: A reliable evaluation item gives reproducible scores with similar populations of students. It is, therefore, independent of the characteristic of individual evaluators. To maintain transparency, evaluative question should test only one option at a time and give the candidates no other option. The evaluation should also adequately reflect the objectives of the teaching concept. The reliability is a measure of the consistency with which the question, test or examination produces the same result under different but both are comparable at different stages.

Validity: A valid evaluation is one which actually tests what is sets out to test for example one which actually measures the behavior described by the objectives under scrutiny. The Pattern of Questions that are intended to check and recall of factual material but which actually test the candidates power of reason or questions with assume a level of pre-knowledge that the candidates do not necessarily process.

Assessment: It's a processes and instruments that are designed to measure the learners achievement, when 
learners are engaged in an instructional program of one or another. Assessment has a powerful meaning than evaluation by a comparing with measurement In its derivation, the word assess means" to sit beside" or "to assist the judge". The faculty uses ratifying a call to assess the social or and personal qualities of students like regularity, punctuality, discipline, the habit of cleanliness, interests and attitude etc.

The grading may be done on a different number of points from three to five points. Marks obtained by each student on the basis of the criteria may be entered in the column provided for them. These marks may be added and the average calculated. The average score could be converted into grades as shown as below:

\begin{tabular}{|c|l|}
\hline Category (Grade) & Marks Obtained by Students \\
\hline A & 3.5 above \\
\hline B & Between 2.5 to 3.5 \\
\hline C & Between 1.5 to 2.5 \\
\hline D & Between 1.5 to 0.5 \\
\hline E & Below 0.5 \\
\hline
\end{tabular}

Table:1

\section{INTERNAL ASSESSMENT}

Internal evaluation and internal curriculum do not necessarily go together. It is possible to assess the performance in an external syllabus by internal means. Internal assessment may be conducted for Institutional level purposes, for example decide who follows which course, or it may be mainly for the information. When internal assessment is used in educational institutions it can do a lot that external assessment cannot. Internal assessment of an internally devised syllabus brigs great freedom choice and opportunities to do such things as field work projects, simulations, drama and practical work. It allows teachers to use their intimate and detailed knowledge of the work of individual pupils across a wide range of activities and objectives over a long period of time.

\section{EXTERNAL ASSESSMENT}

External Assessment is the norm in public examinations. These are set and marked by examination boards and are mainly intended for external users. University authority, employers and in a more general approach the public and their elected representatives in local and central government External examinations are seen as selective courses and their influence on the curriculum as profound. To a limited extent public examinations also use faculty as assessors. A few faculty are involved as paid employees of examination boards in setting examinations but not necessarily do the teacher assess their own students.

\section{TAXONOMICAL MODEL}

It has adopted a three dimensional division of Learning experiences which are classified in various domains listed below:

In Cognitive Domain intellectual component of behavior is represented. It is most important from the view point of education. This domain includes learning objective which deal with recall or recognition of knowledge and development of intellectual activities and skills. This is know as the Knowledge component of educational objectives. Major behaviors in Cognitive Domain are Knowledge, comprehension, Application, Analysis, Synthesis, Evaluation

In Affective domain : This is a higher level of learning at a different level and it has a close relationship with cognitive and behavioral changes. Each affective behavior has a corresponding cognitive behaviors. Major behavior in Affective domain are Receiving, Awareness, Responding, Valuing, Organization, Characterization by a value complex.

In Psychomotor Domain : This model is related to the levels of attainment of neuro-muscular co-ordination. According to this model as the level of co-ordination goes up, the action becomes more refined, speedy and automatic. Major behaviors in Psychomotor Domain are Imitation, Manipulation, Precision, Articulation, Naturalization.

\section{V.STATISTICAL ANALYSIS}

In statistical Techniques of Analysis we can observe the major forms Qualitative and Quantitative data, Continuous and Discrete Data and Primary and Secondary Data.

Qualitative data result from information which has been collected into groups such groups are listed alphabetically or in decreasing frequencies or in some other order for convenience to identify.

Any numerical or quantitative data may be continuous or discrete depending on the nature of the elements or objects being observed. Continuous Data will arise from the continuous variables, in which individual may differ by amounts just approaching zero. The achievement scores of students, though presented in discrete represents any point. The distinction is made on the basis of variable being identified measures. Height is a continuous variable, but number of students would give discrete data.

The data collected by people who are going to make use of the data refers to primary data. Sometimes an investigator may use the data already collected by some one such as the college attendance of students or performances of students in various subjects etc for his her study then the data are secondary data. For using Secondary data it is always useful to know how the data have been collect and 
processed; the accuracy of data; how comparable the data are with other tabulations. Organizing data is very important for construction of bar charts or preparing the analysis of student performance report. To make the study of data easier and suitable it is organized in a proper manner. When a particular data contains a few entries listing of the observations then it is enough for interpreting the data. Every data usually organized into groups called classes and presented in a table which gives the number of observations in each group. We can Assume the arrangement of data in following two ways :
a) Presentation of Data in order
b) Grouping and Tabulation of Data

\section{VI.GRAPHICAL REPRESENTATION}

Histogram : The most common form of graphical representation of data is histogram. For plotting a histogram we need to take a graph paper. The given data values of the variable are taken on the $\mathrm{X}$ - axis and the respective frequencies are taken on the $\mathrm{Y}$ axis.

Bar Diagram : In the given data variable is discrete then it is not possible to construct a histogram. A simple graphical representation is nearby by similar to histogram, know as Bar diagram.

Frequency Polygon : The primary purpose of frequency polygon is to show the shape of the distribution. When two or more frequency distributions ae to be compared the $\mathrm{r}=$ elative frequency polygons are constructed against the same axis.

Cumulative Frequency curve(Ogive) : For plotting a Cumulative Frequency Curve or Ogive, first of all cumulative frequencies against each of the intervals are to be written.

\section{EDUCATIONAL SITUATIONS}

In Educational Curriculum there are lot of stations arise to determine the student statistics in several areas. If we consider one of the data with respect to a class and their marks then Median is used in educational situations as below:

$>$ when incomplete distribution is given,

$>$ when the point dividing the distribution to two equal parts is needed,

$>$ when a distribution is marked, skewed.

Example: 1) Median for the set of scores :

$$
12,17,18,15,20,19 \text {. }
$$

By rearranging the above set the mid term for given order is 17 hence the median of the given distribution is 17.5

Example: 2) Median for 29,18,32,24,19,53,28,49,36,34

\section{is 30.5 (Average of: $5^{\text {th }}$ term 29 and $6^{\text {th }}$ term 32)}

Quartiles deviation Application: Quartiles deviation explains the variables identity as the application process into equal frequency parts. Lower Quartile is Q1, Upper quartile is Q3 and median as Q2. To determined Inner quartile range we use differences between Q3 and Q1.

Formula applicable for Quartile deviation :

$$
\mathbf{Q}=(\mathbf{Q 3}-\mathbf{Q} \mathbf{)}) / 2
$$

Interpretation of Quartile Deviation method : It is convenient to use as a independent extreme value. Comparing to the range it is a good factor for applications. Usually central tendency denotes median in this process and to compute the quartile deviation it is necessary to exist the values of Q, Q1 and Q3

\section{SYMMETRICAL DISTRIBUTIONS}

If the equal frequencies are located in central tendency with same distance in both sides then it can be noted as Symmetrical distribution.

Example:

\begin{tabular}{|l|l|l|l|l|l|l|l|}
\multicolumn{1}{c|}{ C1 } & \multicolumn{1}{c|}{ C2 } \\
\hline Score & 0 & 1 & 2 & 3 & 4 & 5 & 6 \\
\hline Frequency & 2 & 3 & 0 & 1 & 0 & 3 & 2 \\
\hline
\end{tabular}

Table: 2

In this illustration we can observe that cell median is 3 . If we compare both sides frequencies then the order of Cells $\mathrm{C} 1$ : Scores $(0,1,2)$ order is equivalent to $\mathrm{C} 2:(6,5,4)$. Having same Sequences on both sides.

In distributions we can easily observe positive and negative skewness based on the curve end approaches. If the curve end approaches towards left extreme then it is said to be an negative skewed and if it approaches towards right then it is noted as positive skewed.

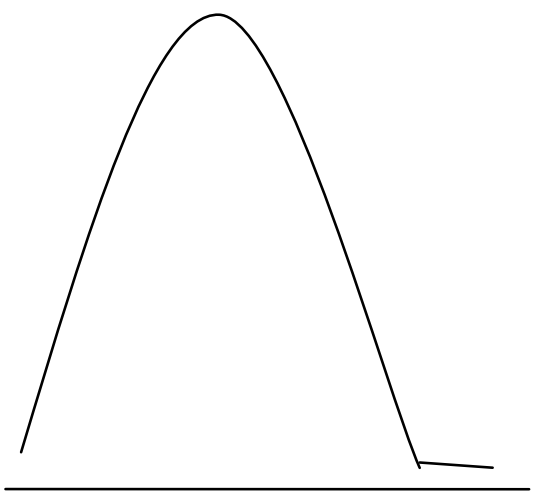

Positive Skewed ( fig.1) 


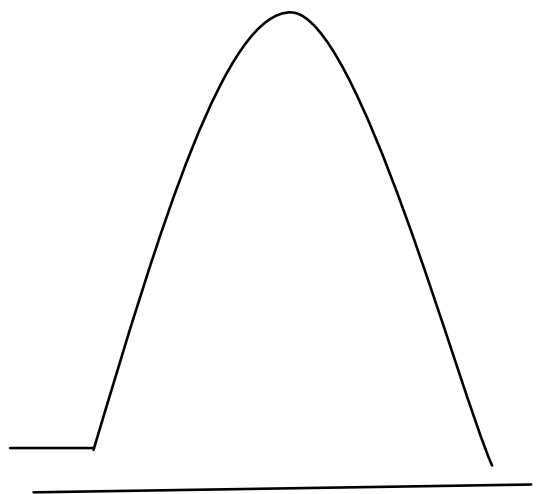

Negatively skewed (fig.2)

\section{NORMAL PROBABILITY CURVES}

In normal probability we can observe that mean, median and mode are coincides at central ordinates. The skewness of one side is similar to other side of the normal distribution. Both the end points are parallel to the base (Xaxis). The end points can be extended left side as negative infinite approach and right side with positive infinite values. Normal probability curves are maintaining equal range on both sides hence can be noted as bilateral distribution.

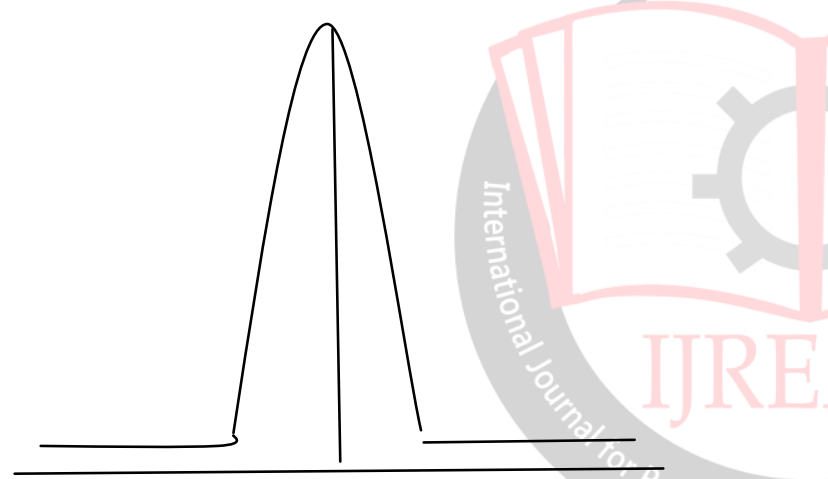

(fig.2)

Mean $=$ Median $=$ Mode

Example :

In a distribution of 1000 stores with $\mathrm{M}=55$ and $\infty=10$.

Then percentage of cases lie between 50 and 65 can be determined in Statistical process as below:

$\mathrm{Z}$ score $=50 ;$ Considering the $\mathrm{Z}$ statistics :

$\mathbf{Z}=(\mathbf{X}-\mathbf{M}) / \boldsymbol{\sigma}$

$\mathrm{Z}=(50-55) 10=-1 / 2$

$\mathrm{Z}$ score $=-0.5 \boldsymbol{\sigma}$

$\mathrm{Z}$ score $=65 ;$ then $\mathrm{Z}$ statistics is

$Z=(65-55) / 10=+1 \sigma$

Through test Statistic(z) tables total percentage of given date $19.15+34.13=53.28$ satisfies the condition .

\section{$\mathbf{x}$ correlation in statistics}

In any Statistical Evaluation correlation means study of relationship or inter-dependence between any two variables. If the change in one variable appears to be accompanied by a change in other variable, then the two variables are said to co-related and this inter dependence is considered as correlation.

When the increase in one variable said $X$ is followed by a corresponding increase in the other variable said Y; the correlation is said to be positive correlation. The positive correlations range from 0 to +1 ; the upper limit is +1 . This perfect positive co-efficient of correlation. The perfect positive correlation specifies in the other. The exact negative correlation indicates that for every unit increase in one variable, there exists its proportional unit decrease in the other.

Null(0) correlation means no relationship between the given two variables $\mathrm{X}$ and $\mathrm{Y}$; that is the change in variable $\hat{\mathrm{Y}}$ is not associated with the change in the other variable $\mathrm{Y}$.

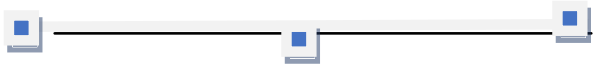

(-1)Negative Correlation $\quad 0$ Positive Correlation(+1)

\section{SPEAR MANS'S CORRELATION}

Spearman's Rank co-efficient of correlation is the operations or measurements of the bivariate variable is based on the ordinal scale in the form of ranks. The rank difference co-efficient of correlation is computed by using the formula :

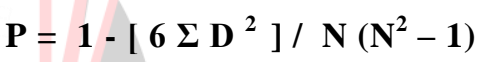

Example: In a State University the following data collected as the scores of 5 students in final year examination in two subjects mathematics and Statistics. Determination of the correlation between the two variables using Rank correlation follows as below :

\section{GIVEN DATE :}

\begin{tabular}{|l|l|l|l|l|l|}
\hline $\begin{array}{l}\text { NUMBER OF } \\
\text { STUDENT }\end{array}$ & $\mathbf{1}$ & $\mathbf{2}$ & $\mathbf{3}$ & $\mathbf{4}$ & $\mathbf{5}$ \\
\hline $\begin{array}{l}\text { MARKS IN } \\
\text { MATHEMATICS }\end{array}$ & $\mathbf{8}$ & $\mathbf{7}$ & $\mathbf{9}$ & $\mathbf{5}$ & $\mathbf{1}$ \\
\hline $\begin{array}{l}\text { MARKS IN } \\
\text { STATISTICS }\end{array}$ & $\mathbf{1 0}$ & $\mathbf{8}$ & $\mathbf{7}$ & $\mathbf{4}$ & $\mathbf{5}$ \\
\hline
\end{tabular}

TABLE $: 3$

\section{CONSIDERING :}

$$
\mathbf{P}=1-\left[\begin{array}{lll}
6 & \Sigma & D^{2}
\end{array}\right] / \mathrm{N}\left(\mathrm{N}^{2}-1\right)
$$

\begin{tabular}{|c|c|c|c|c|c|c|}
\hline Students & Maths & Stats & R1 & R2 & $\begin{array}{r}\text { R2- } \\
\text { R1=D }\end{array}$ & D $^{2}$ \\
\hline 1 & 8 & 10 & 2 & 1 & -1 & 1 \\
\hline 2 & 7 & 8 & 3 & 2 & -1 & 1 \\
\hline 3 & 9 & 7 & 1 & 3 & 2 & 4 \\
\hline 4 & 5 & 4 & 4 & 5 & 1 & 1 \\
\hline 5 & 1 & 5 & 5 & 4 & -1 & 1 \\
\hline $\mathrm{N}=5$ & & & & & $\Sigma \mathrm{D}=0$ & $\mathbf{\Sigma} \mathbf{D}^{2}=8$ \\
\hline
\end{tabular}

Table: 4

$$
P=1-[6 \times 8] / 5\left(5^{2}-1\right)= \pm 0.60
$$


Hence Correlation between two variables $= \pm 0.60$

\section{XII.CONCLUSION}

In Educational Evaluation system the total statistical analysis provides the reports of the students performance as well as the curriculum development rate by the implemented statistical techniques. There is no other process to compare the results of the outcome other than the statistical technique. Hence all the Computations systems, other modern simplification techniques are totally depended as a secondary data. We can say that Statistics is the major resource of the student performance report. Internal assessment, External assessment, campus recruitments, Annual budgets and total investments are simply computed by the Statistical models either in graph representations are in tabular form for easy understanding.

\section{ACKNOWLEDGEMENT}

I am very much thankful to the Vice-Chancellor, Prof. Dr. Dalel Singh, Dean School of Science, Microbiology \& Agriculture and The Registrar of OPJS University for their contribution in publication work. I am extremely grateful to Prof.V.Kannan Former Pro-Vice-Chancellor of University of Hyderabad, who unconditionally gave me his valuable time and guidance in Research Activities. I wish to show my gratitude to my Academic Guru Dr. G. Manohar Reddy Garu and Dr. N. Goutham Rao Garu Management of Swamy Vivekananda Educational Trust, Hyderabad for their motivational thoughts and Support in Academics \& Research activities..

\section{REFERENCES}

[1]. Design and Analysis of Experiments, John Wiley \& Sons, Montgomery,D.C.(1981). New York.

[2]. R.J Statistics for Management and Economics, Mendenhall, W.,Reinmuth J.E and Beaver, R.J Duxbury Press, Belmont CA

[3] The advanced Theory of Statics, Volume 2: Inference and Relationship. Kendall M. and Stuart, A.(1979), Charles Griffin \& Co. Limited, London.

[4] Journals of the American Statistical Association, Two-sided Tolerance Limits for Normal Populations- Some Improvements", Howe, W.G(1969).”64,pages 610-620.

[5] Fundamental Concepts in the Design of Experiments, Hicks,C.R.(1973). Holt, Rinehart and Winston, New York.

[6] Statistical Intervals: A guide for Practitioners, Hahn.G.J and Meeker, W.Q(1991). John Wiley \& Sons,New York.

[7] Introduction to Statistical Analysis, Dixon,W.J and Massey,F.J.(1969). McGraw-Hill,New York

[8] Draper N and Smith,H.,(1981),Applied Regression Analysis, Mc.Graw-Hill,New York.

[9]. Design and Analysis of experiments by Gouglas C.Montgomery - John Wiley \& Sons.

[10]. Draper and Smith : Applied Regression Analysis. 